\title{
ABSOLUTE MAGNITUDES AND COLOURS OF FIELD GIANT BRANCH STARS
}

\author{
H. NECKEL and P. KLAWITTER \\ Hamburg Observatory, F.R.G.
}

\begin{abstract}
Concerning the absolute magnitudes of the giant stars contained in the Mt. Wilson catalogue of Adams et al., the following facts are pointed out:

(1) There is a linear relation between the Mt. Wilson magnitudes and the absolute magnitudes derived on account of the Wilson-Bappu effect (Figure 1). The scale factor is 2.3.

(2) The accuracy of the properly scaled Mt. Wilson magnitudes follows from the scatter in relation (1) and the accuracy of the Wilson-Bappu magnitudes $( \pm 0.6)$. It is $\pm 0 m 6$ (mean error).

(3) The assured reliability of the corrected Mt. Wilson magnitudes allows a subdivision into bright, intermediate and faint giants (Figure 2). The 3 subgroups exhibit different 2-colour relations (Figures $3,4,5)$.

(4) The large number of giant stars present in the Mt. Wilson catalogue allows a trustworthy derivation of the mean absolute magnitudes of giant branch stars (Figure 6) and an estimate of its dispersion as well (Figure 7).
\end{abstract}

Our remarks concern the spectroscopic absolute magnitudes of giant stars given in the Mt. Wilson catalogue published by Adams et al. (1935). It seems to us that the reliability of these magnitudes has been considerably underestimated in the past. (See e.g. the remarks of Blaauw (1963) and of Wilson and Bappu (1957).)

Figure 1 shows the relation between these Mt. Wilson magnitudes $\left(M_{\mathrm{Mw}}\right)$ and those $\left(M_{\mathrm{WB}_{\mathrm{B}}}\right)$ published by Wilson (1959) and Wilson and Bappu (1957). Obviously, there is a common, linear relation for all stars of luminosity classes I to III, with a relatively low scatter. Stars of luminosity class IV are to be excluded because of a separate calibration procedure in the Mt. Wilson work.

The Mt. Wilson magnitudes may be brought into the 'Wilson-Bappu system' using the relation

$$
M_{v}=M_{\mathrm{MW}}^{\text {corr }}=2.3 M_{\mathrm{MW}}-0.1 .
$$

The multiplying factor is close to the rule of thumb value of 2 given by Keenan (1963) for the supergiants, but obviously it should be applied also in the case of the giants. The reason for the scale distortion of the original Mt. Wilson magnitudes has been discussed by Prof. Blaauw this morning.

The mean error of one corrected Mt. Wilson magnitude $M_{\mathrm{MW}}^{\text {corr }}$ follows from the scatter to be seen in Figure 1 and the mean error of the Wilson-Bappu magnitudes. It turns out to be \pm 0.6 , which is just the same accuracy as it is obtainable from the Wilson-Bappu effect (1957).

Figure 2 is a part of the Mt. Wilson HR-diagram. The numerals indicate the numbers of stars present in the Mt. Wilson catalogue for each spectral subclass and each 


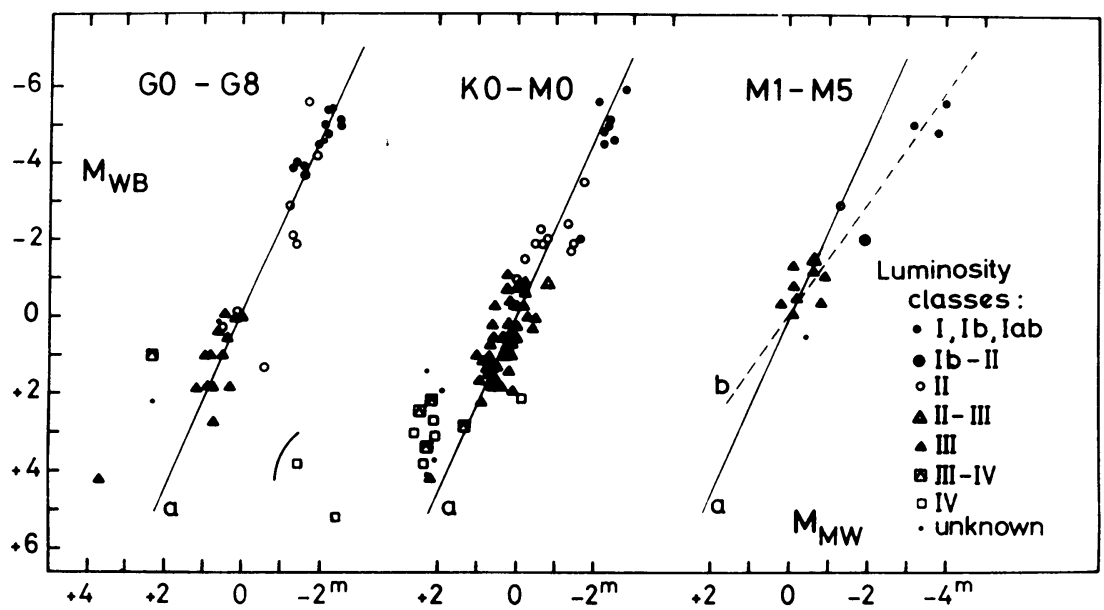

Fig. 1. Relation between absolute magnitudes $M_{\mathrm{Mw}}$ and $M_{\mathrm{W} \text { B }}$.

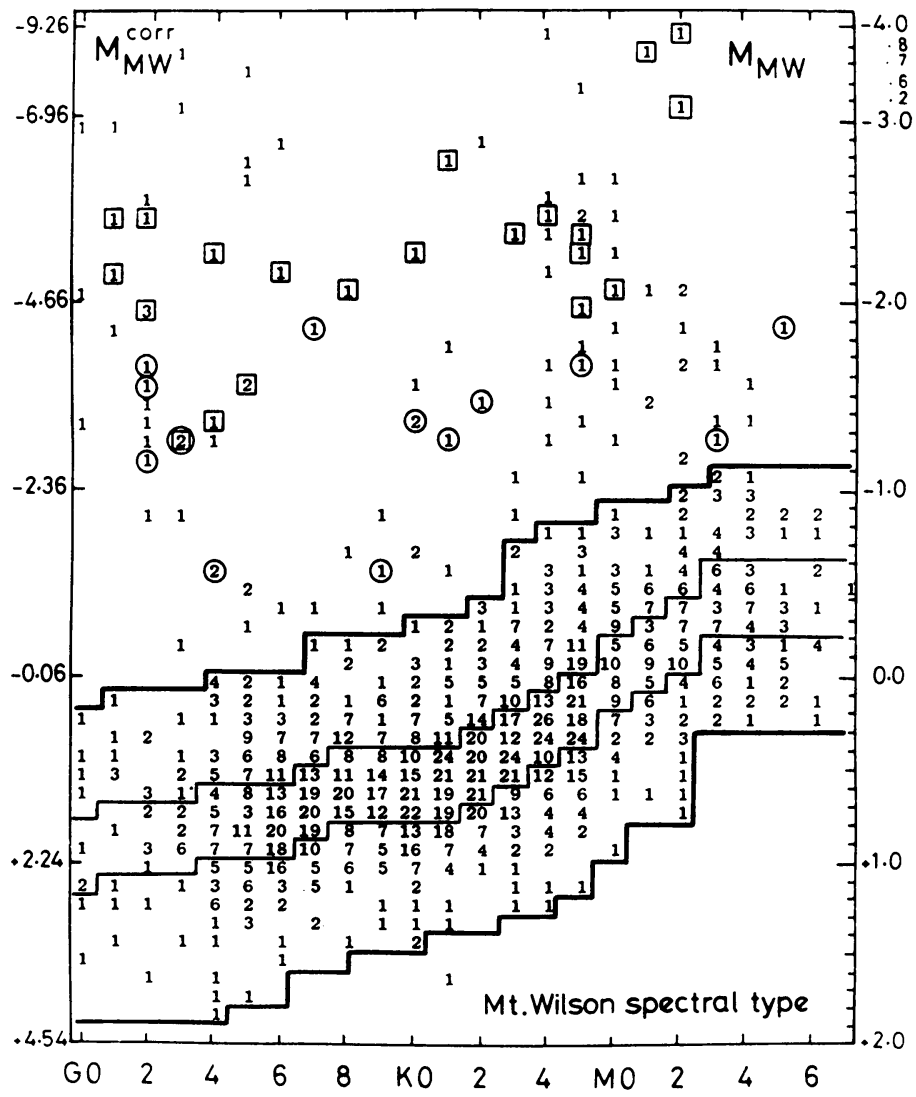

Fig. 2. Upper right sector of the Mt. Wilson HR-diagram. 
tenth of (uncorrected) magnitude. The left-hand scale gives the corrected Mt. Wilson magnitudes.

Numerals within squares indicate stars of luminosity class I, numerals within circles indicate stars of luminosity class II.

The stars between the heavy lines are considered to be giant branch stars, which is not necessarily identical with luminosity class III. The weak lines divide the giant branch into bright, intermediate and faint giants $(\alpha, \alpha \beta, \beta)$. This subdivision was

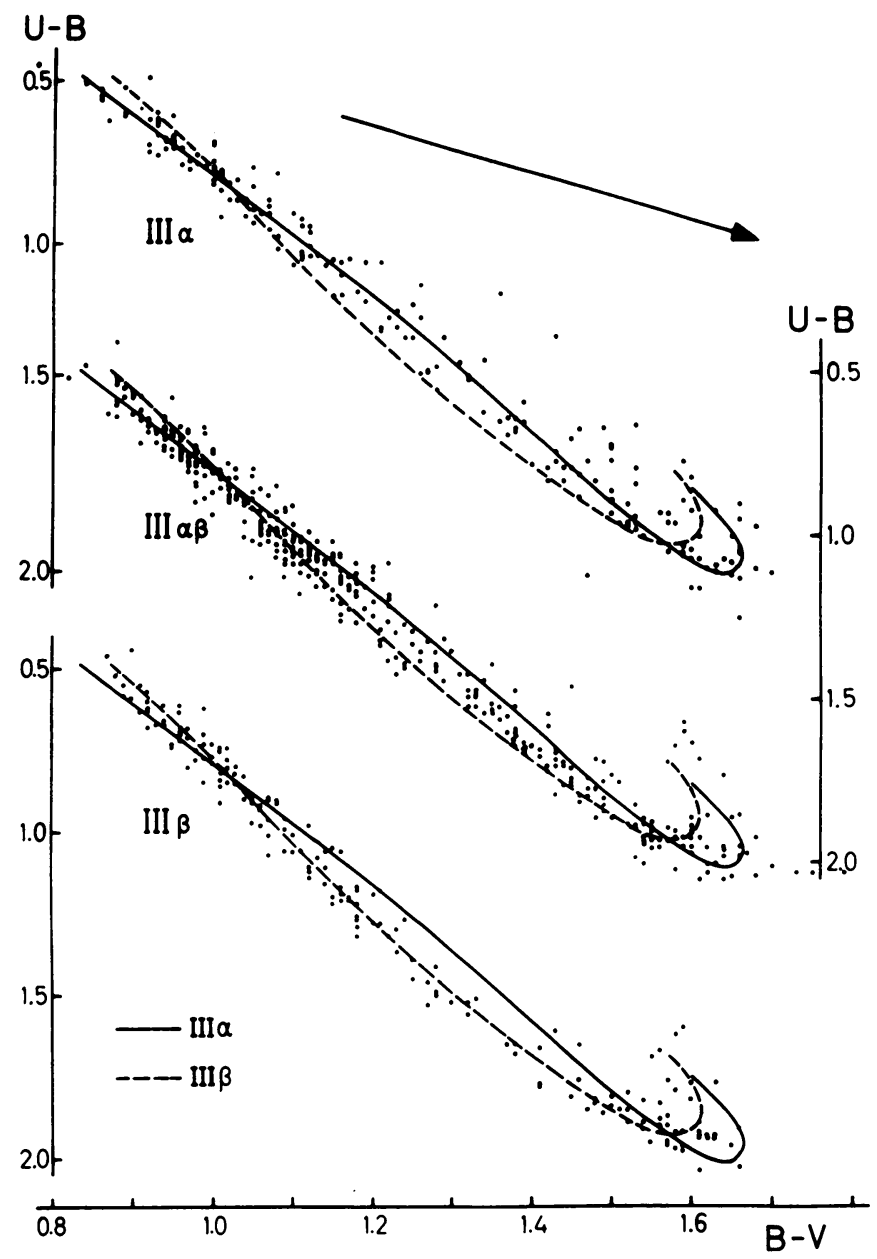

Fig. 3. Two-colour diagrams for 3 luminosity groups of giant stars.

made purely arbitrarily, but intending to get about the same numbers of stars in each subbranch.

Figure 3 stresses the significance of this subdivision: Here we plotted the 2-colour 
diagrams separately for the 3 subgroups. The colours were taken from the photometric catalogue published by Blanco et al. (1970). The solid and the broken lines mark the mean relations for the upper and lower subgroup respectively. The arrow marks the reddening line. Apparently, the 3 subgroups occupy, on the average, different areas in the HR-diagram.

Figure 4 gives the mean colours for the different spectral classes. The crosses are based on all stars of the specific spectral class, which are included in both the Mt. Wilson catalogue and the photometric catalogue of Blanco et al. These values correspond to those given by FitzGerald (1970) for luminosity class III stars. Filled and open circles mark the mean colours for the bright $(\alpha)$ and faint $(\beta)$ members of each spectral class.

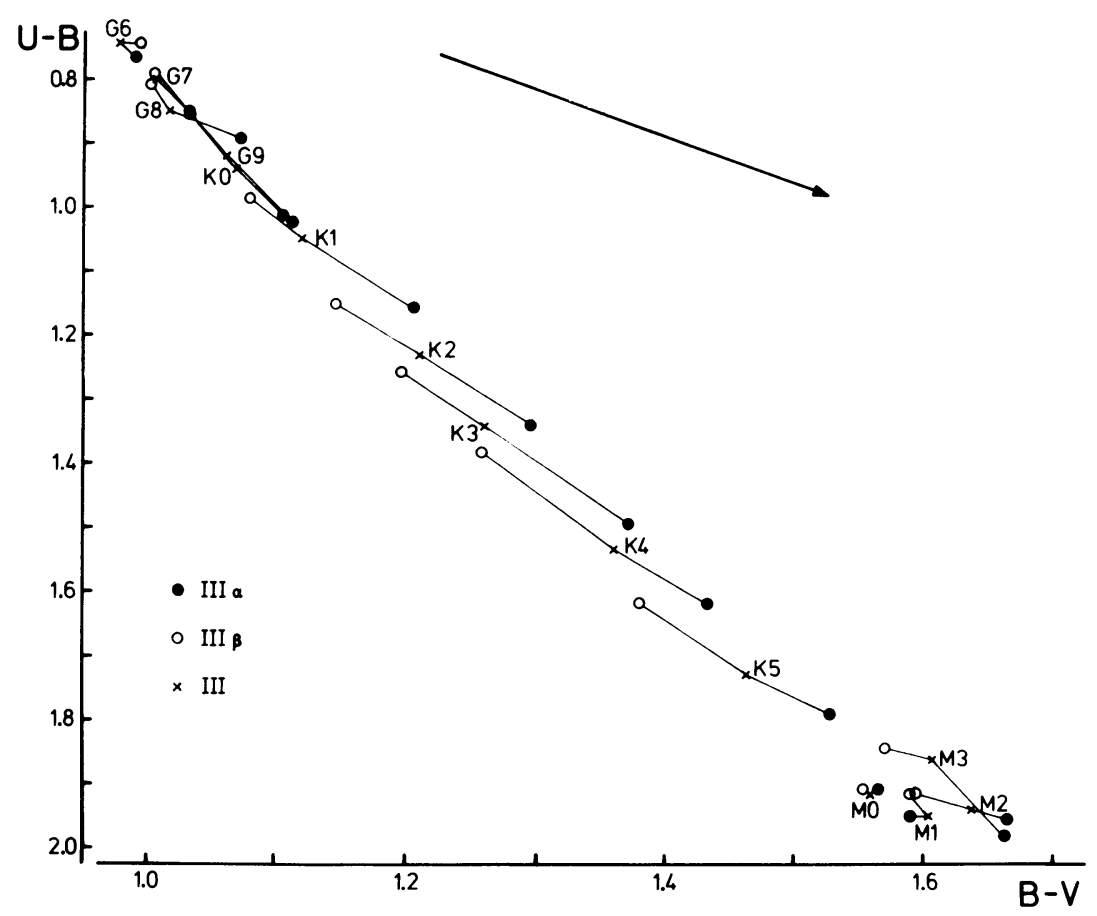

Fig. 4. Mean colours of giant stars.

Figure 5 shows for 4 selected spectral classes the distribution of individual stars around that mean 2-colour-relation, which is based on all giant stars (connecting the crosses in Figure 4).

Figures 4 and 5 reveal that, on the average, the absolutely brighter giants are redder than the fainter objects, in both colours. It has been proven that this effect is not caused by interstellar reddening: There is not traceable dependency of the colour on the distance. Most of the stars are brighter than 6th magnitude and are within $100 \mathrm{pc}$. 
(Remark during the discussion: There may be circumstellar reddening, which is dependent on absolute magnitude.)

Figure 6 shows the mean values of the corrected Mt. Wilson absolute magnitudes for the fields giants as a function of spectral type. A reduction to equal volume of space was not made yet, but the appropriate correction will be in the order of a tenth of a magnitude only. For comparison, the results or compilations of several other authors are given also.

In Figure 7 we have plotted the number of giants and supergiants present in the Mt. Wilson catalogue versus the distance $M_{v}-\bar{M}_{v}$ from the mean giant branch (step-

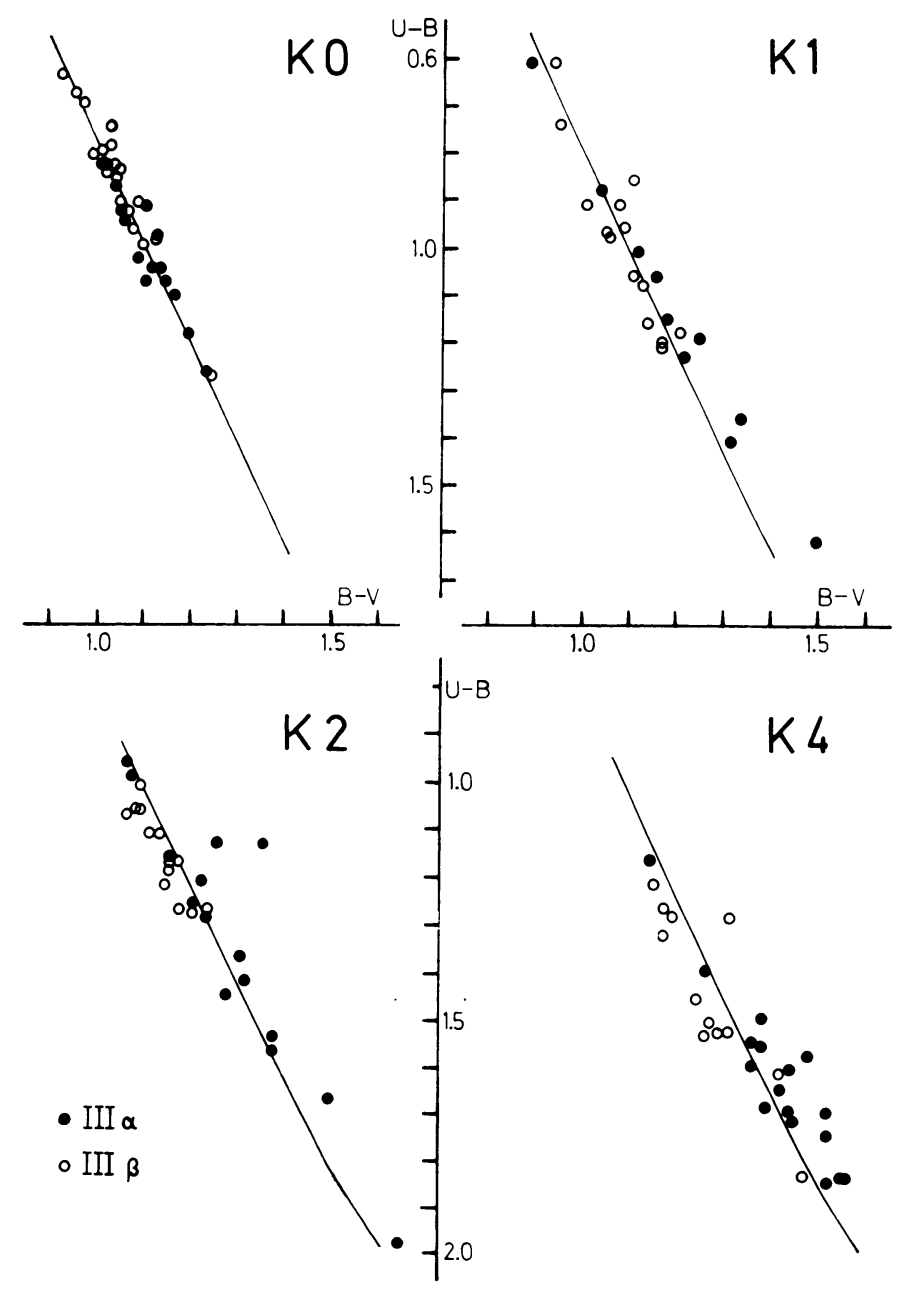

Fig. 5. Two-colour diagrams for stars of 4 selected spectral classes. 


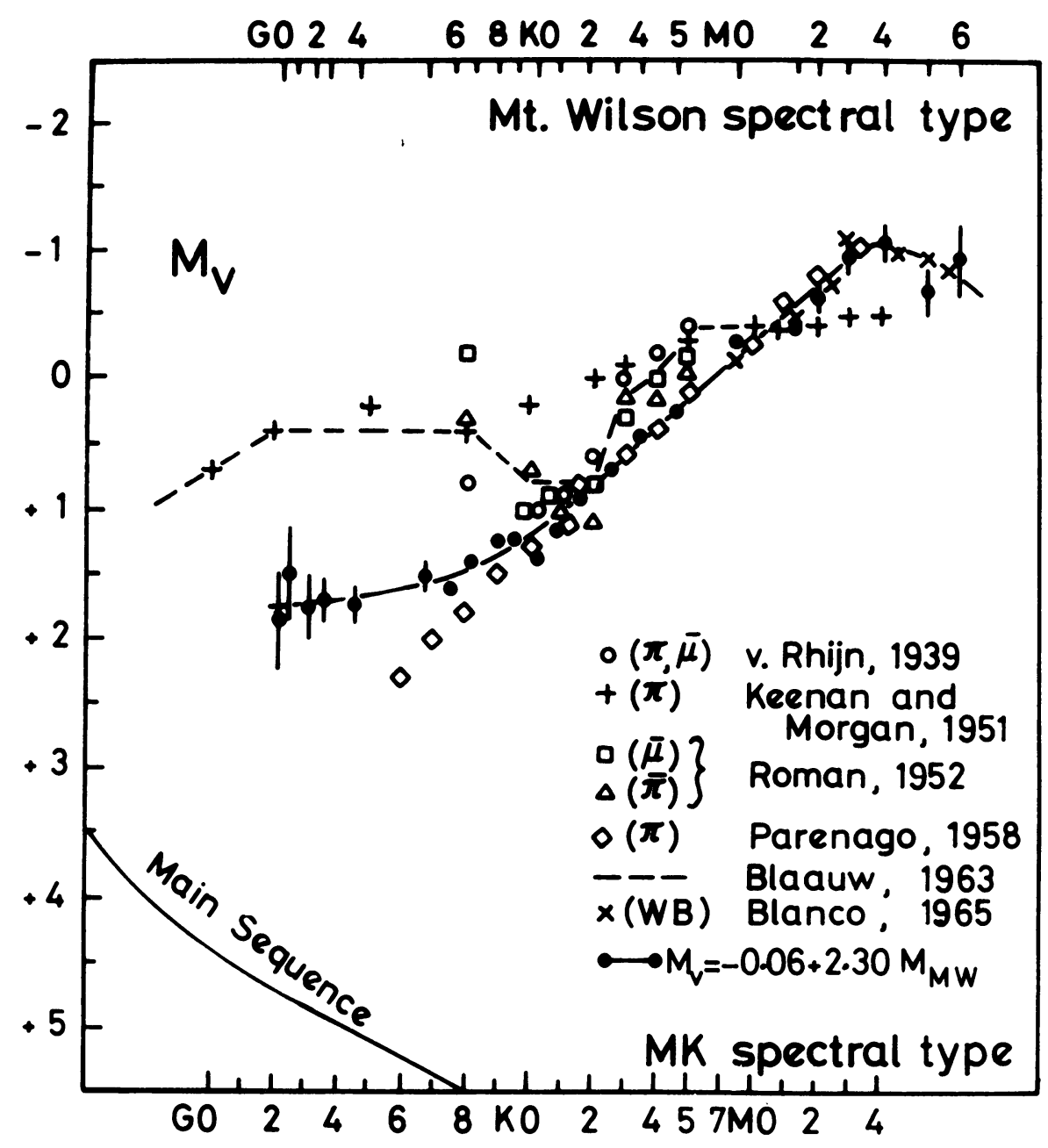

Fig. 6. Mean absolute magnitudes of giant branch stars.

like, full line; $M_{v}=$ corrected Mt.Wilson magnitude). The standard deviation is \pm 0.73 , which is only slightly larger than the observational error of \pm 0 . 6 , which was obtained from the scatter in Figure 1. This would indicate that the cosmic scatter of the absolute magnitudes of field giant branch stars cannot be considerably larger than about \pm 0 . 5 . The dashed and the dash-pointed lines are Gaussian profiles with $\sigma= \pm 0.73$ (observed distribution) and \pm 0.44 ('true' dispersion).

\section{References}

Adams, W. S., Joy, A. H., Humason, M. L., and Brayton, A. M.: 1935, Astrophys. J. 81, 187.

Blaauw, A.: 1963, Stars and Stellar Systems 3, 383. 


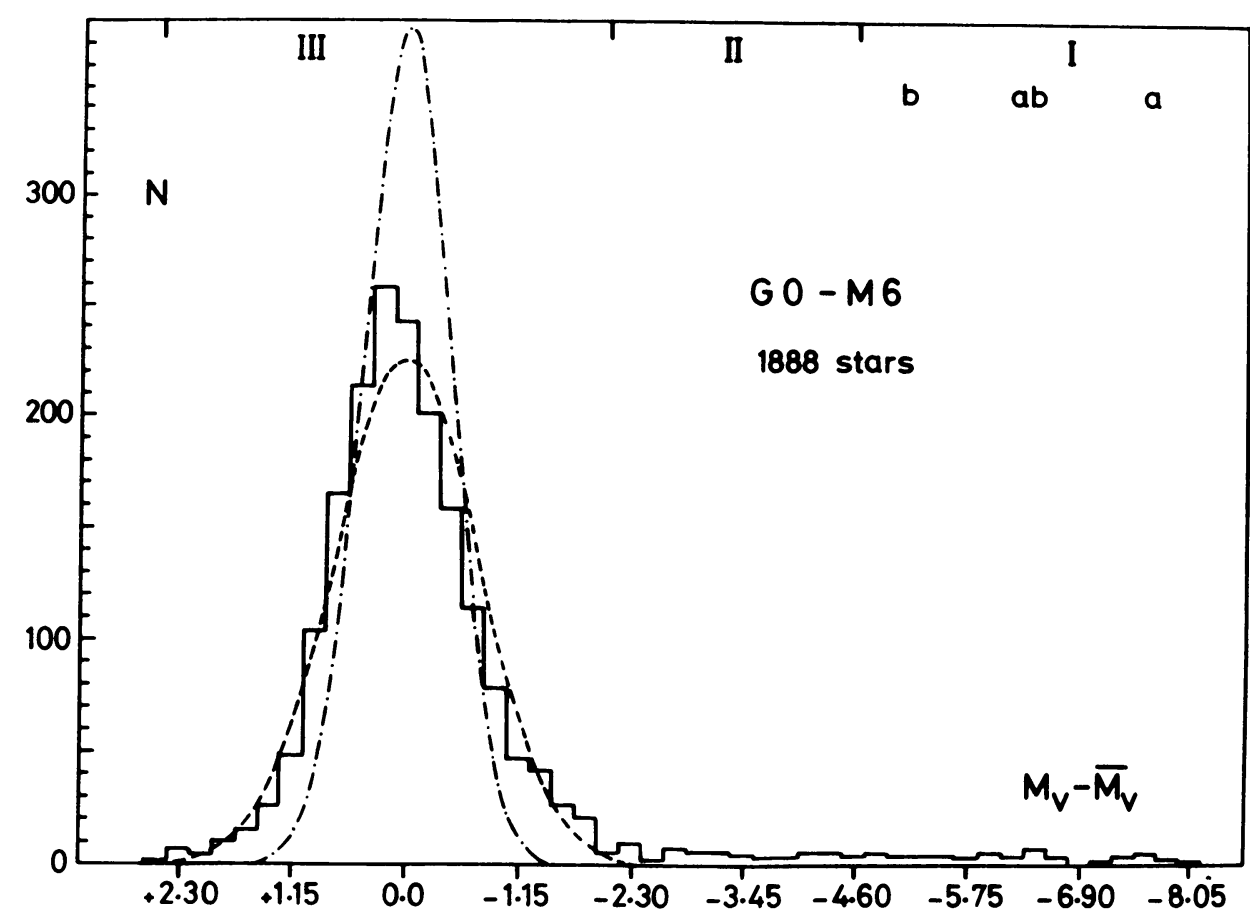

Fig. 7. Distribution of giant and supergiant stars around mean position of the giant branch.

Blanco, V. M.: 1965, Stars and Stellar Systems 5, 241.

Blanco, V. M., Demers, S., Douglas, G. G., and FitzGerald, M. P.: 1970, Photoelectric Catalogue, Publ. U.S. Naval Obs., 2nd series, Vol. 21.

FitzGerald, M. P.: 1970, Astron. Astrophys. 4, 234.

Keenan, P. C.: 1963, Stars and Stellar Systems 378.

Keenan, P. C. and Morgan, W. W.: 1951 in J. A. Hynek (ed.), Astrophysics, p. 17.

Parenago, p.p.: 1958, Soviet Astron. A. J. 2, 151.

Rhijn, van P. G.: 1939, Publ. Capteyn Astron. Lab. Groningen, No. 49.

Roman, N. G.: 1952, Astrophys. J. 116, 122.

Wilson, O. C.: 1959, Astrophys. J. 130, 499.

Wilson, O. C. and Bappu, M. K. V.: 1957, Astrophys. J. 125, 661.

\section{DISCUSSION}

Pecker: The difference between your III $\alpha, \beta$ classes could be due (and one of your slides is clearly pointing to this interpretation) to circumstellar reddening in the case of $\mathbf{G}-\mathbf{M}$ giants where mass ejection could well be a general phenomenon.

Steinlin: Neckel's Figure 7 cannot show distinctly the supergiant classes II to Ia because it shows the difference $M_{v}$ (III) to $M_{v}$ (II) etc., and this varies slightly with spectral type. So plotting all spectral types in one diagram will necessarily smear out the higher luminosity classes.

Blaauw: Is it correct to say that the photometric differences between your classes III $\alpha$ and III $\beta$ indicate properties already brought out in the Mt. Wilson spectroscopic classification, independent of the re-scaling of the absolute magnitudes?

Neckel: Yes, it is correct. But these photometric differences confirm the distinctness of the Mt. Wilson magnitudes with regard to the giants. 